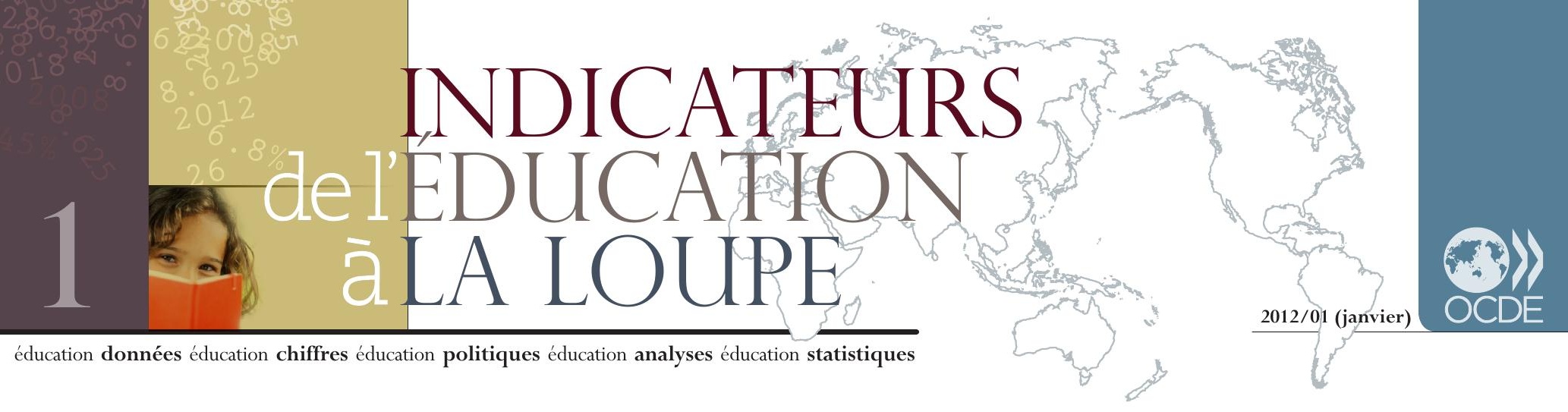

\title{
Quel est l'impact de la crise économique mondiale sur les individus en fonction de leur niveau de formation?
}

- Entre 2008 et $\mathbf{2 0 0 9}$, le taux de chômage a augmenté à tous les niveaux de formation dans les pays de l'OCDE, et ce de façon particulièrement inquiétante chez les individus non titulaires d'un diplôme de fin d'études secondaires.

- En 2009, dans les pays de l'OCDE, le taux d'emploi moyen était nettement plus élevé parmi les diplômés de l'enseignement tertiaire (ou supérieur), signe d'une meilleure adéquation de leurs compétences avec la demande sur le marché de l'emploi.

- Entre 2008 et 2009, l'avantage salarial en faveur des diplômés de l'enseignement tertiaire est resté important dans les pays de l'OCDE. Dans certains d'entre eux, l'écart de revenus s'est même creusé entre les diplômés de l'enseignement tertiaire et les individus dont le niveau de formation est inférieur au deuxième cycle de l'enseignement secondaire.

L'impact de la crise économique sur les individus se mesure à l'aune de leur niveau de formation.

À I'heure où la crise financière dans le monde occidental, les taux de chômage bloqués à des niveaux élevés et la croissance économique à l'arrêt dans de nombreux pays contribuent à créer un climat d'incertitude pour 2012, il paraît opportun de dresser un bilan des retombées sur la population de la crise économique mondiale qui a commencé en 2008. Dans les pays membres de l'OCDE ainsi que dans les pays partenaires, ce bilan est clair : la récession a affecté bien plus durement les individus les moins qualifiés.

Au plus fort de la crise, les individus Alors que la crise amorçait un départ fulgurant en 2008 puis se les moins qualifiés étaient nettement confirmait en 2009, le taux de chômage des 25-64 ans a augmenté plus susceptibles de se trouver sans emploi... de façon généralisée dans les pays de l'OCDE. Pourtant, l'impact fut bien plus prononcé chez les individus n'ayant pas atteint le deuxième cycle de l'enseignement secondaire. Parmi ce groupe, le taux de chômage a augmenté de 2.8 points de pourcentage, en moyenne, entre 2008 et 2009, passant de $8.7 \%$, un niveau déjà élevé, à $11.5 \%$. Les hommes et les femmes de ce groupe ont été particulièrement touchés en Espagne, en Estonie, aux États-Unis et en Irlande, où le taux de chômage des individus dont le niveau de formation est inférieur au deuxième cycle du secondaire a fait un bond de 5 points de pourcentage, voire davantage, entre ces deux années.

L'impact de la crise s'est révélé moins brutal chez les 25-64 ans diplômés du deuxième cycle du secondaire ou de l'enseignement post-secondaire non tertiaire. Dans les pays de l'OCDE, leur taux de chômage a augmenté de presque 2 points de pourcentage, en moyenne, entre 2008 et 2009, passant de $4.9 \%$ à $6.8 \%$. Toutefois, dans plusieurs pays, notamment en Espagne, en Estonie, en Irlande, en République slovaque et en Turquie, le taux de chômage des individus bénéficiant de ce niveau de formation a pratiquement atteint, voire dépassé, les $10 \%$. Les États-Unis ont échappé de peu à ce constat.

En revanche, même au plus fort de la crise mondiale, les diplômés de l'enseignement tertiaire (ou supérieur) s'en sont bien mieux sortis que les individus moins qualifiés. Globalement, le taux de chômage des 25-64 ans bénéficiant de ce niveau de formation n'a augmenté que de 1.1 point de pourcentage entre 2008 et 2009, passant de $3.3 \%$ à $4.4 \%$, soit une augmentation correspondant à moins de la moitié du pic de chômage constaté chez les individus n'ayant pas atteint le deuxième cycle du secondaire. 
Ainsi, le taux de chômage des diplômés de l'enseignement tertiaire n'a pas dépassé la barre des $5 \%$ en 2009 dans 24 des 34 pays de I'OCDE et au Brésil (pays partenaire). Par ailleurs, en 2009 toujours, le taux de chômage de ce groupe n'a excédé les $8 \%$ que dans deux pays de l'OCDE : I’Espagne et la Turquie.

Variation du taux de chômage, selon le niveau de formation (2008-09)

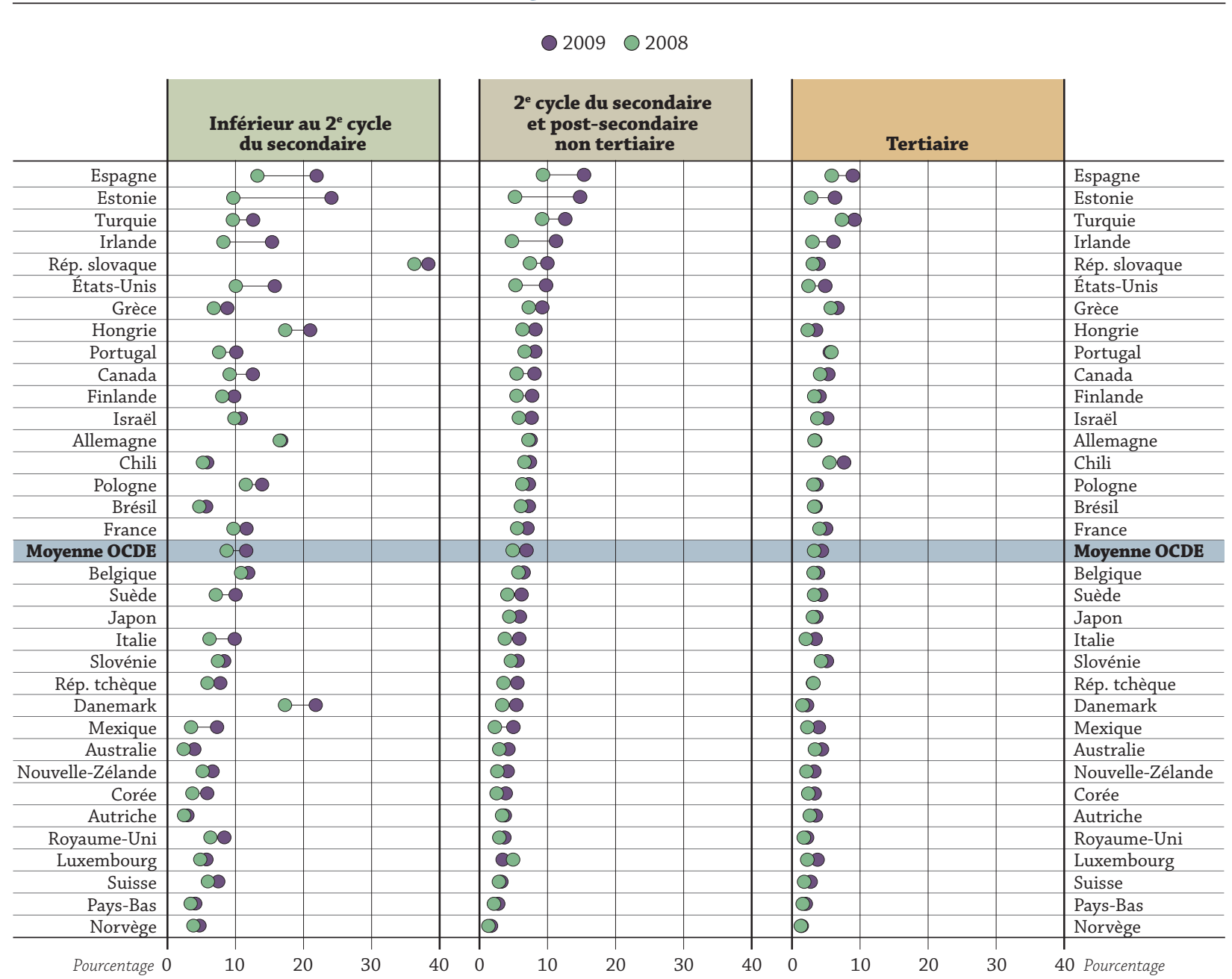

Les pays sont classés par ordre décroissant du taux de chômage en 2009 des diplômés du deuxième cycle de l'enseignement secondaire ou de l'enseignement post-secondaire non tertiaire. Source : Regards sur l'education 2011 : Les indicateurs de l'OCDE, Indicateur A7 (www.oecd.org/edu/eag2011).

... tandis que les diplômés de l'enseignement tertiaire étaient bien plus susceptibles d'avoir un emploi stable.

Les chiffres de l'emploi font la preuve de l'avantage compétitif certain dont bénéficient sur le marché du travail les actifs les plus qualifiés, et ce que l'on soit ou non en temps de crise. En moyenne, dans tous les pays de l'OCDE, $83.6 \%$ des 25-64 ans diplômés de l'enseignement tertiaire occupaient un emploi en 2009 , contre $56.0 \%$ des 25-64 ans dont le niveau de formation est inférieur au deuxième cycle du secondaire - soit un écart de 27.6 points de pourcentage. En Hongrie, en Pologne, en République slovaque et en République tchèque, cet écart dépasse les 40 points de pourcentage, tandis qu'en Corée, en Islande et en Nouvelle-Zélande, il représente 15 points de pourcentage, voire moins. 
Pourcentage d'actifs occupés dans la population âgée de 25 à 64 ans, selon le niveau de formation (2009)

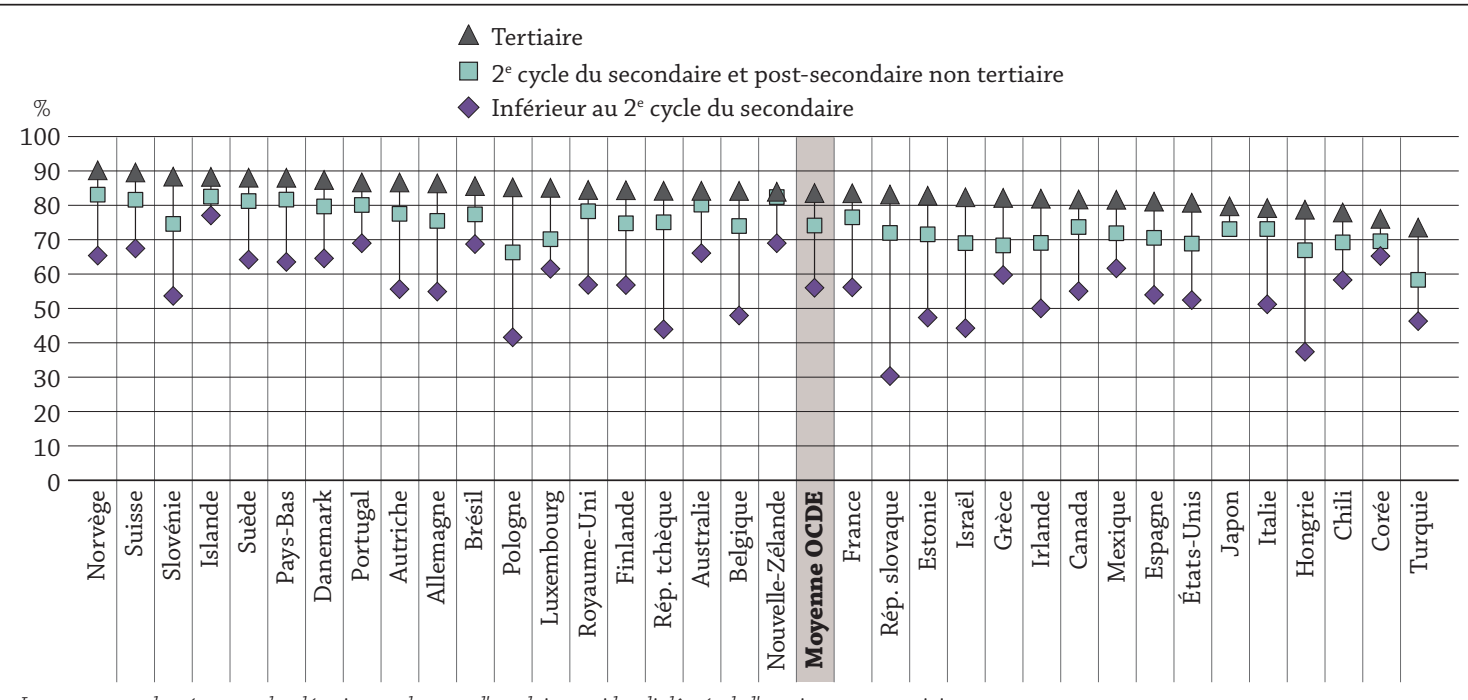

Les pays sont classés par ordre décroissant du taux d'emploi parmi les diplômés de l'enseignement tertiaire.

Source : Regards sur l'education 2011 : Les indicateurs de l'OCDE, Indicateur A7 (www.oecd.org/edu/eag2011).

Lecture du graphique

Ce graphique montre la corrélation positive entre emploi et formation. Dans tous les pays, les diplômés du deuxième cycle de l'enseignement secondaire ou post-secondaire non tertiaire sont plus susceptibles de travailler que ceux dont le niveau de formation est inférieur, et les diplômés de l'enseignement tertiaire sont plus susceptibles de travailler que les diplômés du deuxième cycle de l'enseignement secondaire. Toutefois, l'importance de l'avantage que procurent des diplômes de ces niveaux varie selon les pays.

\section{Taux d'emploi et taux de chômage}

Contrairement à une idée reçue, taux d'emploi et taux de chômage ne sont pas directement liés. Le taux d'emploi se rapporte au nombre d'individus occupant un emploi en pourcentage de la population en âge de travailler. Le taux de chômage mesure, quant à lui, le nombre d'individus sans emploi, en recherche active d'emploi et disponibles pour commencer à travailler, en pourcentage de la population active civile et au cours d'une période de référence donnée.

De la même manière, les diplômés de l'enseignement tertiaire étaient bien plus nombreux à occuper un emploi que les diplômés du deuxième cycle du secondaire ou de l'enseignement post-secondaire non tertiaire. En moyenne, dans tous les pays de I'OCDE, 74.2\% des 25-64 ans bénéficiant de ces niveaux de formation occupaient un emploi en 2009, soit un écart de 9.5 points de pourcentage par rapport au taux d'emploi des diplômés de l'enseignement tertiaire. Si les variations du taux d'emploi d'un pays à l'autre s'expliquent par de nombreux facteurs, tout particulièrement par la participation des femmes à la population active, le meilleur taux d'emploi des individus plus qualifiés est la preuve d'une meilleure adéquation de leurs compétences à la demande sur le marché de l'emploi, y compris en temps de crise.
L’avantage salarial dont bénéficie un employé type diplômé de l'enseignement tertiaire s'est maintenu pendant la crise.

\section{Avantages et désavantages salariaux, selon le niveau de formation (2008-2009) \\ Population totale des 25-64 ans}

(par rapport aux revenus du travail moyens des individus ayant atteint le deuxième cycle du secondaire ou le niveau post-secondaire non tertiaire)

\begin{tabular}{l|c|c} 
Moyenne des pays* & 2008 & 2009 \\
\hline Tertiaire & $+56 \%$ & $+57 \%$ \\
\hline Inférieur au $2^{\mathrm{e}}$ cycle du secondaire & $-23 \%$ & $-23 \%$ \\
\hline
\end{tabular}

*La moyenne inclut les 14 pays de l'OCDE dont les données sont disponibles pour les deux années.

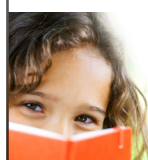


